\title{
Targeted deletion of numb and numblike in sensory neurons reveals their essential functions in axon arborization
}

\author{
Eric J. Huang, ${ }^{1,4,7}$ Huashun Li, ${ }^{2,3}$ Amy A. Tang, ${ }^{1,4}$ Amanda K. Wiggins, ${ }^{1,4}$ Rachael L. Neve, ${ }^{5}$ \\ Weimin Zhong, ${ }^{6}$ Lily Y. Jan, ${ }^{2,3}$ and Yuh Nung Jan ${ }^{2,3}$ \\ Departments of ${ }^{1}$ Pathology and ${ }^{2}$ Physiology and ${ }^{3}$ Howard Hughes Medical Institute, University of California San Francisco, \\ San Francisco, California 94143, USA; ${ }^{4}$ Pathology Service, Veterans Administration Medical Center, San Francisco, \\ California 94121, USA; ${ }^{5}$ Departments of Psychiatry and Genetics, Harvard Medical School, McLean Hospital, Belmont, \\ Massachusetts 02178, USA; ${ }^{6}$ Department of Molecular, Cellular and Developmental Biology, Yale University, \\ New Haven, Connecticut 06520, USA
}

\begin{abstract}
Mouse Numb homologs antagonize Notch1 signaling pathways through largely unknown mechanisms. Here we demonstrate that conditional mouse mutants with deletion of numb and numblike in developing sensory ganglia show a severe reduction in axonal arborization in afferent fibers, but no deficit in neurogenesis. Consistent with these results, expression of Cre recombinase in sensory neurons from numb conditional mutants results in reduced endocytosis, a significant increase in nuclear Notch1, and severe reductions in axon branch points and total axon length. Conversely, overexpression of Numb, but not mutant Numb lacking $\alpha$-adaptin-interacting domain, leads to accumulation of Notch1 in markedly enlarged endocytic-lysosomal vesicles, reduced nuclear Notch1, and dramatic increases in axonal length and branch points. Taken together, our data provide evidence for previously unidentified functions of Numb and Numblike in sensory axon arborization by regulating Notch1 via the endocytic-lysosomal pathways.
\end{abstract}

[Keywords: Numb; Numblike; Notch1; sensory neurons; axon arborization; endocytosis]

Supplemental material is available at http://www.genesdev.org.

Received August 4, 2004; revised version accepted October 28, 2004.

One of the major events in the developing nervous system is for the neural progenitor cells to generate a wide variety of differentiated neurons that serve a multitude of physiological functions. Genetic analyses in Drosophila have indicated that a series of events controls the development of external sensory organ in the peripheral nervous system (Jan and Jan 2001). This includes solicitation of the proneural clusters by basic helix-loop-helix genes, the achaete-scute complex or atonal, and cell fate specification by Notch, Delta, and numb. While the cellular components of the Drosophila external sensory organs and the mammalian peripheral nervous system are seemingly different, accumulating evidence has indicated that some of the molecular mechanisms controlling their development may be highly conserved across species. For instance, analyses of mouse mutants demonstrate that Mash-1, mouse homolog of Drosophila achaete-scute genes, is required for the development of

${ }^{7}$ Corresponding author.

E-MAIL: ejhuang@itsa.ucsf.edu; FAX (415) 750-6947.

Article published online ahead of print. Article and publication date are at http://www.genesdev.org/cgi/doi/10.1101/gad.1246005. sympathetic neurons, whereas neurogenin-1 and neurogenin-2, homologs of atonal, cooperatively regulate the differentiation of a full repertoire of sensory neurons in the cranial and trunk sensory ganglia (Johnson et al. 1990; Guillemot et al. 1993; Ma et al. 1998, 1999; Anderson 1999; Perez et al. 1999). Similar to the misexpression data in fly, ectopic expression of neurogenins in cells within dermomyotome of chick embryos activates expression of neuron-specific genes, indicating that neurogenins provide the essential intrinsic cues for sensory neuron development (Perez et al. 1999).

The mammalian numb homolog, mouse Numb, interacts with the intracellular domain of Notch1 and can rescue the numb mutant phenotype in Drosophila (Zhong et al. 1996). During cortical neurogenesis, Numb is asymmetrically localized to the apical membrane of the dividing progenitor cells in the periventricular zone, suggesting that Numb may control asymmetric cell division in this cell population (Zhong et al. 1996). Consistent with this model, deletion of floxed numb (numb ${ }^{\text {flox }}$ ) using the ubiquitous $\beta$-actin-Cre or EIIaCre results in early embryonic lethality with precocious neuronal production in the developing forebrain and severe defects in 
neural tube closure (Zhong et al. 2000; Zilian et al. 2001). Interestingly, deletion of numb ${ }^{\text {flox }}$ using nestin-Cre, which targets recombination in the majority of neural progenitor cells as early as embryonic day 8.5 (E8.5) does not lead to any detectable deficits during embryogenesis or in adult mice, suggesting that loss of numb may be compensated by another mammalian numb homolog, numblike (nb1) (Petersen et al. 2002). Indeed, both mRNA and protein of $\mathrm{nbl}$ are present in the developing nervous system, especially in post-mitotic neurons of the cortical plate and peripheral nervous system (Zhong et al. 1997). Moreover, deletion of numb using nestin-Cre in the $n b l$ null background results in severe thinning of the entire axis of neural tube due to a severe depletion of progenitor cells in the nervous system (Petersen et al. 2002). Although these data underscore the essential, yet redundant, roles of numb and $n b l$ in regulating proliferation of the early neural progenitor cells, removal of Numb and $\mathrm{Nbl}$ at later stages results in drastically different phenotypes in cortical neurogenesis (Li et al. 2003; Petersen et al. 2004). Deletion of numb using the Emx1-Cre (Emx1cDKO) causes hyperproliferation in the neural progenitors with severe disorganization in the cerebral cortex ( $\mathrm{Li}$ et al. 2003). In contrast, deletion of numb using a D6-Cre (D6-cDKO), which is expressed in cortical progenitors after the firstborn neurons are generated, leads to depletion of neural progenitors and marked thinning of the cortex (Petersen et al. 2004). While the exact cause of this discrepancy remains unclear, several possible mechanisms have been proposed, including the effects from trace amount of Numb in the mutants, timing of numb deletion by Cre recombinase at different developmental stages, and the potential effects of Numb loss of function on the Cajal-Retzius cells or radial glial cells (Castaneda-Castellanos and Kriegstein 2004). It is important to note that targeted deletion of mouse Lethal giant larvae homolog, Lg11, results in a loss of polarity in neural progenitors, loss of asymmetric localization of Numb, and a hyperproliferation phenotype similar to that seen in Emxl-cDKO mutants (Klezovitch et al. 2004).

In contrast to the tremendous interests of Numb and $\mathrm{Nbl}$ in maintaining the progenitor cells in the periventricular zones, much less is known about their roles in sensory neurogenesis. Several lines of evidence indicate that Notch signaling pathways may regulate sensory neuron development in vertebrates. First, expression of Notch in avian neural crest stem cells causes an irreversible loss of neuronal capacity and instructively promotes glial differentiation (Morrison et al. 2000). Second, the avian homolog of Numb shows a stage-dependent, asymmetrical segregation in mitotic cells within the nascent dorsal root ganglion (DRG) during embryogenesis, suggesting that Numb may control asymmetric cell division in the developing sensory nervous system (Wakamatsu et al. 2000). Finally, numb conditional mutants $\left(\right.$ numb $\left.b^{\text {neo-lox }}\right)$, when crossed with an ubiquitous deleter EIIaCre, reportedly show a nearly complete absence of sensory neuron markers NeuroD and NF160 in DRG and an up-regulation of glial marker ErbB3 at E10.5, suggest- ing that Numb is required for cell fate determination in sensory neurogenesis (Zilian et al. 2001). However, the early embryonic lethality due to severe vascular defects causes a generalized malformation in these mutant mice and raises the question that the deficits in sensory neurogenesis may not be a direct consequence of deletion in numb (Zilian et al. 2001).

To directly test the roles of Numb and $\mathrm{Nbl}$ in mouse sensory neurogenesis and to circumvent the embryonic lethality, we deleted numb in the migrating neural crest and in nascent DRG using the Wnt1Cre transgenic line (Jiang et al. 2000). Our data showed that loss of Numb and $\mathrm{Nbl}$ in the sensory ganglia had no detectable effect on generation and differentiation of sensory neurons. In contrast, our data uncovered a novel, cell-autonomous function for Numb and $\mathrm{Nbl}$ in regulating sensory axon arborization through the regulation of Notch1 via the endocytic-lysosomal pathways.

\section{Results}

Sensory ganglion-specific targeting of numb in nbl null background leads to perinatal lethality

To investigate the roles of Numb in mouse sensory neuron development, we generated conditional numb mutant mice in which numb was deleted in sensory ganglia using Wnt1Cre (Fig. 1). By using the ROSA26R mice as a reporter (Soriano 1999), we showed that Wnt1Cre generated recombination in the migrating neural crest cells as early as E9.5 (Fig. 1A,B, highlighted by lacZ+ cells). At E10.5 and E11.5, the coalesced DRG showed an intense lacZ staining, and at E15.5, both the neuronal and nonneuronal cell populations continued to show intense positive staining (Fig. 1C-E). In addition, Wnt1Cre transgene also generated recombination in the dorsal part of spinal cord, where most interneurons showed positive staining for lacZ (Fig. 1D).

Sensory ganglion-specific deletion of numb was generated by crossing homozygous numb conditional mutants $($ numb flox $/$ numb flox mutants, designated as $f n b / f n b)$ with Wnt1Cre mice that carry one copy of the numb flox allele (Wnt1Cre/+;fnb/+). Examinations of Wnt1Cre/+;nb/fnb embryos at E11.5 and E15.5 showed no detectable Numb protein in the sensory ganglia (Fig. 1F-I). Mice homozygous for fnb allele and carrying one copy of Wnt1Cre transgene (Wnt1Cre/+;fnb/fnb) were born with normal Mendelian ratio and survived through adulthood with no difference in body weight, reproductive ability, or behaviors compared with those of littermates. The lack of any conspicuous neurological defect in Wnt1Cre/+;fnb/fnb mice suggested that there might be functional redundancy between Numb and $\mathrm{Nbl}$ in sensory neurogenesis. To test this hypothesis, we generated mice that carried the Wnt1Cre transgene and were homozygous for numb flox and $n b l$ alleles (designated as Wnt1Cre/+; $n b / f n b ; n b 1^{-/-}$). In contrast to Wnt1Cre/+;fnb/fnb mice, Wnt1Cre/+;fnb/ $f n b ; n b 1^{-1-}$ mutant mice were perinatally lethal and exhibited severe neurological deficits, including hyperextension of extremities, inability to ride into prone posi- 
Huang et al.

Figure 1. Conditional deletion of numb in the developing sensory ganglia. $(A-E)$ The recombination pattern of Wnt1Cre in trunk neural crest and developing sensory ganglia was examined by crossing Wnt1Cre transgenic mice with ROSA26 mice. The regions where recombination occurred showed intense lacZ staining. $(A, B)$ Wnt1Cre recombination pattern at E9.5 was revealed in whole-mount staining $(A)$ and tissue sections at the trunk level (indicated by arrows in $A) .(B, C)$ lacZ $^{+}$cell in the dorsal spinal cord (arrowhead in $B$ ) and in scattered cells in the migratory paths of trunk neural crest (arrow). A few lacZ ${ }^{+}$cells are identified around dorsal aorta (DA) at the presumptive site for future sympathetic ganglion (arrow in $B)$. (C) Whole-mount lacZ staining in E10.5 embryo showed intense lac $Z^{+}$cells in

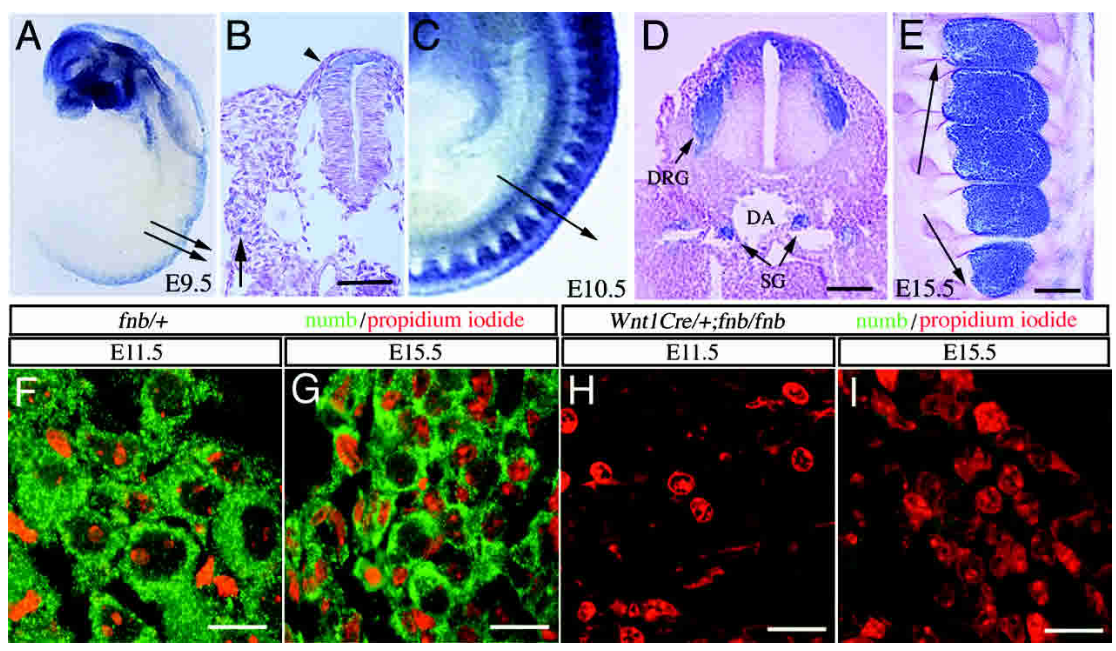
the coalesced DRG. $(D) \mathrm{LacZ}^{+}$cells were also present in the sympathetic ganglia (SG) adjacent to the DA. (E) At E15.5, essentially every cell within the dorsal root ganglion is positive for lacZ. $(F-I)$ No detectable amount of Numb protein was identified in the sensory ganglia of Wnt1Cre/+;fnb/fnb at E11.5 (H) and E15.5 (I). Bars: B,D,E, $50 \mu \mathrm{m} ; F-I, 20 \mu \mathrm{m}$.

tion, reduced responses to external stimuli, and lack of spontaneous suckling activity.

Development of sensory neurons is unaffected

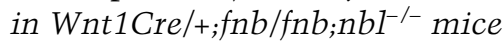

To delineate the neurological deficits in Wnt1Cre/+;nb/ $f n b ; n b 1^{-/-}$mice, we first examined the process of neurogenesis in sensory ganglia. By using antibodies specific for Trk receptors and parvalbumin (representing TrkC neurons), we found no difference in the number of sensory neuron subtypes between control $\left(f n b /+; n b l^{+/-}\right)$ mice and Wnt1Cre/+;fnb/fnb;nbl-/- mutants (Fig. 2). Comparable numbers of $\operatorname{TrkA}^{+}, \mathrm{TrkB}^{+}$, or parvalbumin ${ }^{+}$ neurons were detected in the lumbar DRG at post-natal day 0 (P0) (Fig. 2A-C,E-G,I). The morphology of sensory afferent axons in the dorsal roots showed no difference in epon-embedded ultrathin sections (Fig. 2D,H,I). Taken together, data in Figure 2 indicate that removal of endogenous Numb using Wnt1Cre in the background of $n b 1^{-1-}$ mutants has no effect on the generation of sensory neurons and its differentiation into three distinct subtypes. Furthermore, since the number of axons in the dorsal roots are similar in both control and Wnt $1 \mathrm{Cre} /+; f n b / f n b /$ $n b 1^{-/-}$mutants, loss of Numb and $\mathrm{Nbl}$ does not affect the initial formation of sensory axons.

\section{Reduction in branching of sensory afferent fibers} in Wnt1Cre/+;fnb/fnb;nbl ${ }^{-/-}$mutants

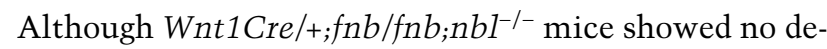
tectable defects in sensory ganglia and spinal interneurons, we found a consistent reduction in the size of dorsal funiculus in the spinal cord of Wnt1Cre/+; fnb/fnb; $n b 1^{-/-}$mutants ( $n=4$ at E15.5 and P0) (Fig. 3, cf. A,C and $E, G)$. The basic organization of dorsal funiculus of the

Figure 2. Neuronal differentiation in the sensory ganglia is not affected in Wnt1Cre/+; $f n b / f n b ; n b 1^{-/-}$mice. Differentiation of TrkA, TrkB, and parvalbumin-positive neurons in the DRG of control $(A-C)$ and Wnt1Cre/ $+; f n b / f n b ; n b 1^{-/-}(E-G)$ mice at PO. $(I)$ The total numbers of $\operatorname{TrkA} \mathrm{A}^{+}$neurons were determined at the level of $\mathrm{L} 1$, $\mathrm{TrkB}^{+}$neurons were determined at $\mathrm{L} 2$, and parvalbu$\mathrm{min}^{+}$neurons were determined at the level of L3. $(D, H, I)$ The number of sensory afferent axons in the dorsal roots of control $(D)$ and conditional mutant $(H)$ mice was not significantly different at the thoracic level. At least three animals are used for counting in each group. Numbers represent mean \pm SEM.

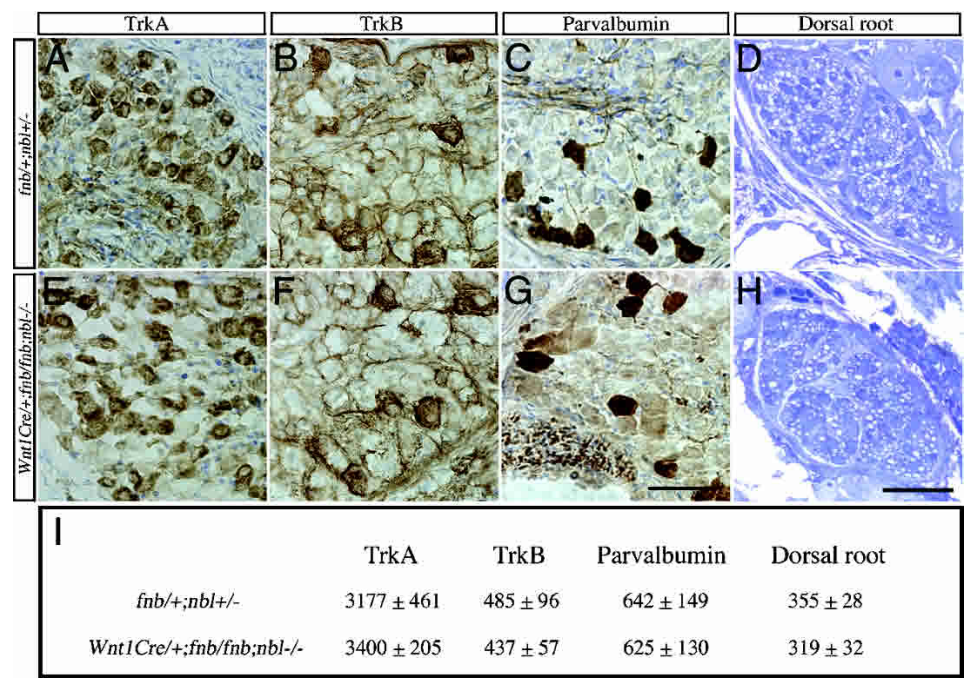




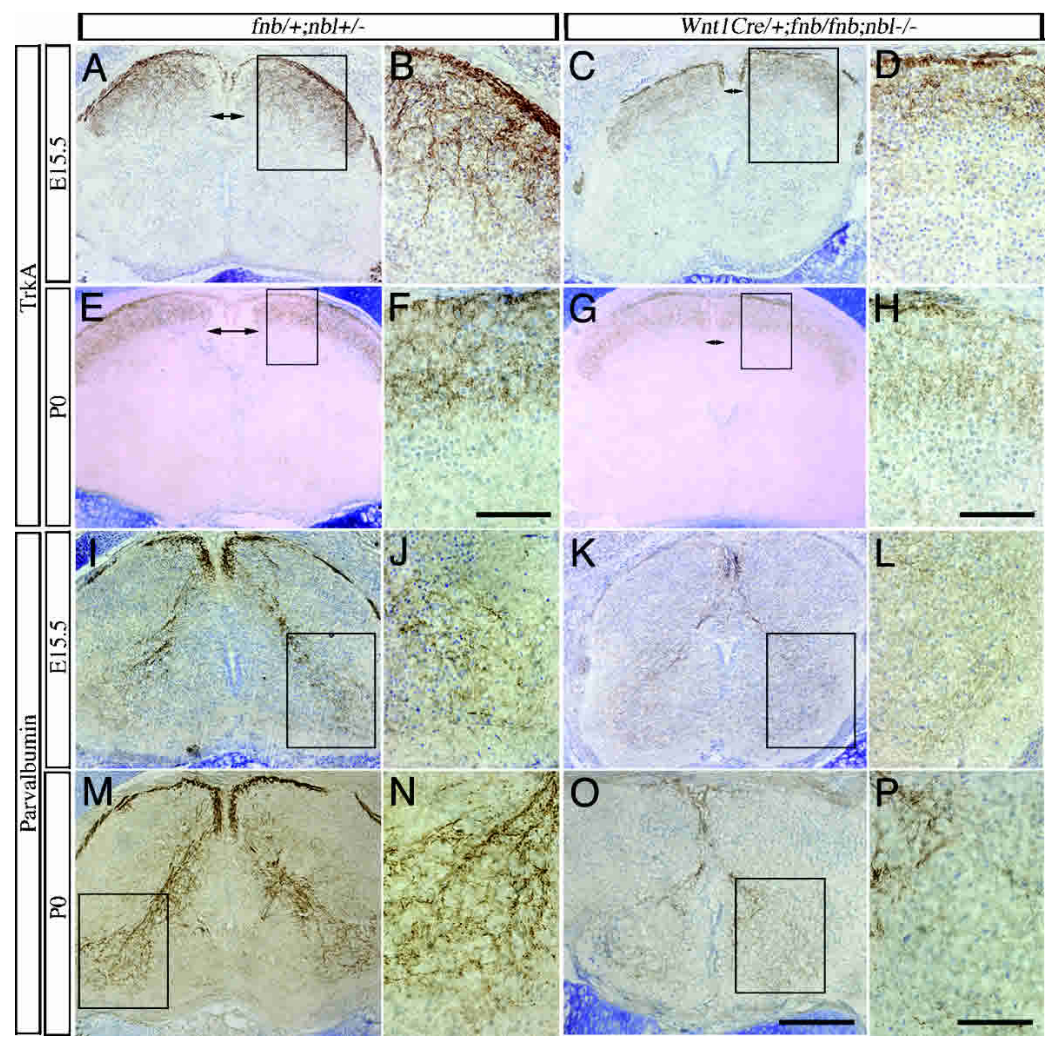

Figure 3. Defects in nociceptive and proprioceptive innervation in the spinal cord of Wnt1Cre/ $+; f n b / f n b ; n b l^{-/-}$mice. Nociceptive sensory afferent fibers, highlighted by the TrkA antibody, are examined at E15.5 and P0. $(A-D)$ At E15.5, exuberant ingrowth of $\mathrm{TrkA}^{+}$fibers is identified in the developing dorsal horns of control spinal cord $(A, B)$. Some of the TrkA ${ }^{+}$fibers extend into the intermediate layers of the dorsal horn. In contrast, the intensity of $\mathrm{TrkA}^{+}$fibers is much reduced in the spinal cord of Wnt1Cre/+; $n b / f n b ; n b 1^{-/-}$mice. Note the reduction in the size of dorsal funiculus (DF, double arrows) in mutant spinal cords. Comparisons are made at the similar of levels of the spinal cord. $(E-H)$ By P0, TrkA ${ }^{+}$fibers form a compact layer of innervation at the dorsal horn of control mice, whereas the staining intensity of TrkA ${ }^{+}$ fibers continues to be reduced in the mutants. Compare the staining intensity in panels $F$ and $H$. Proprioceptive fibers are labeled using the antibody that recognizes parvalbumin. $(I-L)$ As early as E15.5, parvalbumin ${ }^{+}$fibers form compact bundles in the dorsal roots (arrows) and dorsal funiculus (arrowheads) and project into the ventral horn of the spinal cord $(I, J)$. In contrast, only sparse number of parvalbumin ${ }^{+}$fibers are identified in the dorsal funiculus of the mutant spinal cord $(K, L)$. The control spinal cord at P0 shows intense parvalbumin $^{+}$fibers in the Ia afferent projection to the ventral spinal cord. In mutant spinal cord; however, the number of parvalbumin ${ }^{+}$fibers remains significantly reduced and only spare parvalbumin $^{+}$fibers are identified in the ventral horn. Bars: $F, H, 25 \mu \mathrm{m} ; O, 200 \mu \mathrm{m} ; P, 50 \mu \mathrm{m}$. spinal cord consists of a major contribution from ascending and descending collateral branches of primary sensory afferent fibers and axons of the propriospinal neurons, and a minor component from several descending pathways from the brain (Brown 1981; Willis and Coggeshall 1991). The consistent reduction in the size of dorsal funiculus, in the absence of detectable deficits in dorsal roots (Fig. 2D,H,I), suggested that loss of Numb and $\mathrm{Nbl}$ may influence branching of sensory axons.

To test this hypothesis, we examined the central projections of nociceptive and proprioceptive sensory afferent fibers using cell type-specific antibodies TrkA and parvalbumin, respectively. At E15.5, the TrkA ${ }^{+}$sensory afferents showed intense staining at the dorsal root entry zone and projected exuberant branches to the superficial layers of the dorsal horn, some of which extended into the intermediate layers (Fig. 3A,B). In contrast, the intensity of TrkA $\mathrm{A}^{+}$fibers was significantly reduced in both the dorsal root entry zone and the dorsal horn of mutant mice, with most TrkA ${ }^{+}$fibers limited to the superficial layers of the dorsal horn (Fig. 3C,D). While the TrkA ${ }^{+}$ fibers in control mice continued to show intense staining in the substantia gelatinosa at $\mathrm{P0}, \mathrm{TrkA}^{+}$fibers in mutant mice showed a conspicuous reduction in staining intensity, albeit less prominent compared with E15.5 (Fig. 3E-H). Since TrkA staining intensity in the cell body of sensory neurons was comparable in control and mutant mice (Fig. 2A,E), these data suggested that loss of $\mathrm{Numb}$ and $\mathrm{Nbl}$ in sensory neurons resulted in a reduction in the branching of nociceptive afferent axons. To examine if loss of Numb and $\mathrm{Nbl}$ had a more general effect on collateral branching in sensory neurons, we investigated the projection of proprioceptive afferent axons using parvalbumin antibody. Since motor neurons are located outside of the Wnt1Cre recombination domains (Fig. 1D), any effect on the projection of the Ia afferents most likely is due to loss of Numb and Nbl in sensory neurons. In agreement with previously published results (Arber et al. 2000), we found that the parvalbumin antibody highlighted classic Ia sensory afferent pathway, which innervated motor neurons in the ventral horn of the spinal cord (Fig. 3I,J,M,N). In E15.5 control mice, the parvalbumin $^{+}$sensory afferents were present in the dorsal funiculus and projected to motor neurons in the ventral horn (Fig. 3I,J). At P0, the proprioceptive afferents form complex terminal arborization in the ventral horn, in which many of the collateral branches contained intense and punctate parvalbumin ${ }^{+}$immunoreactivity that has been previously shown to be located in the boutons of en passant synapses (Brown 1981). In contrast, the dorsal funiculus of Wnt1Cre/+; $f n b / f n b ; n b 1^{-/-}$mutants showed a dramatic reduction in the staining intensity for parvalbumin, and the size of the Ia afferent fiber bundles was reduced (Fig. 3K,L,O,P). 
Huang et al.

\section{Cell-autonomous effects of Numb and $\mathrm{Nbl}$ on sensory axon branching}

The reduction in TrkA ${ }^{+}$and parvalbumin ${ }^{+}$sensory afferents in the spinal cord of Wnt1Cre/+; fnb/fnb; $n b l^{-/-}$mutants strongly suggested that $\mathrm{Numb}$ and $\mathrm{Nbl}$ were required for axonal branching in sensory neurons. To examine axonal growth and branching defects at the single cell level, we cultured sensory neurons from trigeminal ganglion and DRG of perinatal mice of various genotypes in the presence of NGF or NT3, and induced deletion of numb flox allele by infecting neurons with herpes simplex virus that carried codon-improved Cre recombinase (HSV-iCre) (Shimshek et al. 2002). Recent data indicate that NGF and NT3, respectively, support nocieptive and proprioceptive sensory neurons that show distinct axonal morphology in culture (Patel et al. 2000; Markus et al. 2002). Hence, this approach provides a convenient means to investigate the effect of Numb and Nbl loss of function at the single cell level.

At 50-100 moi (multiplicity of infection), HSV-iCre achieved high level of expression in most neurons with abundant Cre recombinase protein accumulating inside neuronal nuclei (Fig. 4A,B). At $72 \mathrm{~h}$ after infection, there was no detectable Numb immunoreactivity in $f n b / f n b$; $\mathrm{nbl}^{-1-}$ neurons expressing iCre (Fig. 4B, arrow). By using this approach, we examined the effect of Numb and $\mathrm{Nbl}$ loss of function in axonal arborization with emphases on the number of branch points, maximal axon length, and total axon length (Fig. 4). In NGF-responsive neurons, the number of branch points in wild-type, $\mathrm{fnb} /+; \mathrm{nbl}^{+/-}$, $f n b /+; n b 1^{-/-}$, and $f n b / f n b ; n b 1^{+/-}$sensory neurons infected by HSV-iCre showed no significant difference. In contrast, the number of branch points in $f n b / f n b ; n b 1^{-/-}$neurons showed $>50 \%$ reduction compared with that of wild type (Fig. 4C). Consistent with the absence of defect in the dorsal roots of Wnt1Cre/+;fnb/fnb;nbl ${ }^{-/-}$mutants (Fig. 2), there was no reduction in the maximal axonal length of HSV-iCre-infected $f n b / f n b ; n b l^{-/-}$neurons. In contrast, total axon length in $f n b / f n b ; n b l^{-/-}$neurons was significantly reduced (Fig. $4 \mathrm{C}$ ). Loss of Numb and $\mathrm{Nbl}$ had similar effects on the axonal branching and total axon length of NGF and NT3-responsive neurons from both trigeminal ganglion and DRG (Fig. 4C,D). Taken together, these results were consistent with the sensory

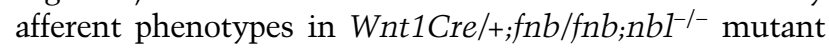
mice and supported that $\mathrm{Numb}$ and $\mathrm{Nbl}$ regulated axonal branching via a cell-autonomous mechanism.

Loss of numb and $\mathrm{nbl}$ reduces endocytosis and leads to a modest increase in nuclear Notch1

The sensory afferent phenotype in Wnt1Cre/+;fnb/fnb; $n b 1^{-/-}$mutant mice and the reduction in axon branch points and total axon length in Cre-expressing $f n b / f n b$; $\mathrm{nbl}^{-/-}$neurons are reminiscent of the effects of constitutively active Notch1 (Notch1 intracellular domain or Notch1 ICD) in regulating neurite outgrowth in cortical neurons (Berezovska et al. 1999; Sestan et al. 1999; Red-
Figure 4. Loss of Numb and $\mathrm{Nbl}$ results in reduced axonal length and arborization in sensory neurons in culture. $(A)$ Expression of Cre recombinase in control sensory neurons did not affect the level of endogenous Numb protein. $(B)$ In contrast, Cre recombinase effectively abolished the expression of Numb in $f n b / f n b ; n b l^{-/-}$neurons (arrow), whereas the level of endogenous Numb protein remained unchanged in neurons without Cre. $\left(A^{\prime}, B^{\prime}\right)$ Camera lucida drawings of axons in control neurons grown in the presence of NGF or NT3 $\left(A^{\prime}\right)$ and $f n b / f n b ; n b 1^{-/-}\left(B^{\prime}\right)$ neurons upon expression of Cre recombinase. Bars: $A-B, 10 \mu \mathrm{m} .(C)$ For NGF-responsive neuron, cultured from either trigeminal ganglion (TG) or dorsal root ganglia (DRG), loss of Numb and $\mathrm{Nbl}$ reduced axon branch points and total axon length, without affecting the maximal axon length. Compared with wild-type neurons, the number of axon branch points and branch index were significantly reduced in $f n b / f n b ; n b 1^{-/-}$neurons, but not in $f n b /+; n b 1^{-/-}$or $f n b / f n b ; n b 1^{+/-}$neurons. ${ }^{\star} p<0.01 ;{ }^{\star}{ }^{\star} 0.01<p<0.05$. The maximal axon length, however, was not significantly different between wild-type and $f n b / f n b$; $n b 1^{-1-}$ neurons. $(D)$ Loss of Numb and $\mathrm{Nbl}$ also had similar effects on the axon branch points and total axon length in NT3-responsive neurons from both TG and DRG. ${ }^{\star} p<0.01 ;{ }^{\star} 0.01<p<0.05$. Paired Student's $t$-test was performed for each statistical analyses, except the axon branch point panel of $C$, where one way ANOVA was used followed by a Tukey multiple comparison test.
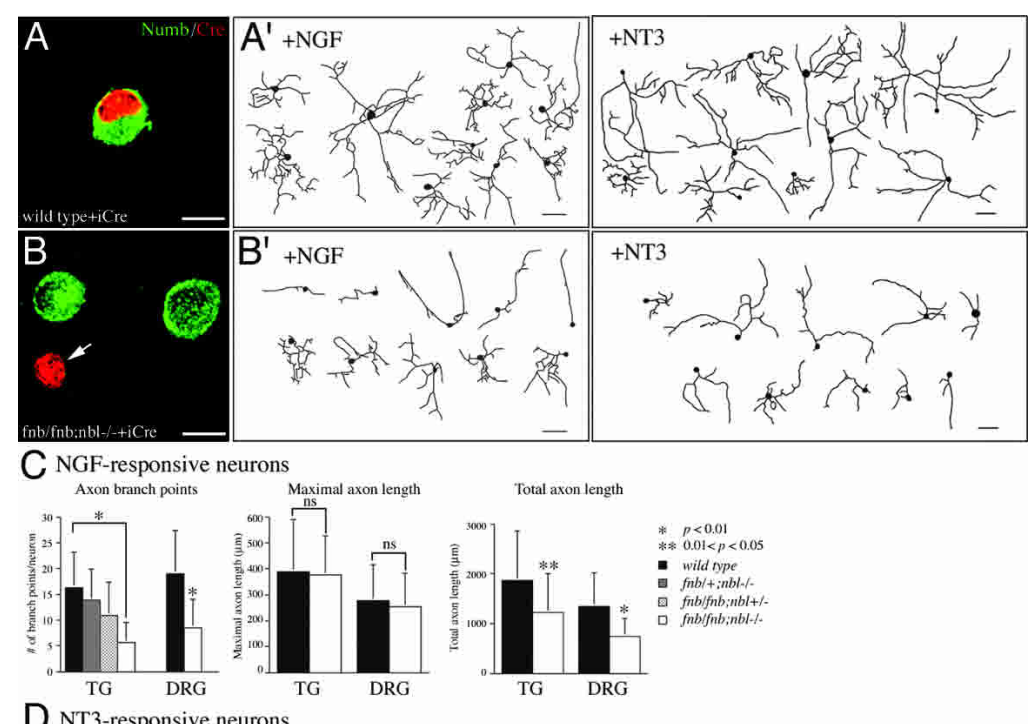

D NT3-responsive neurons
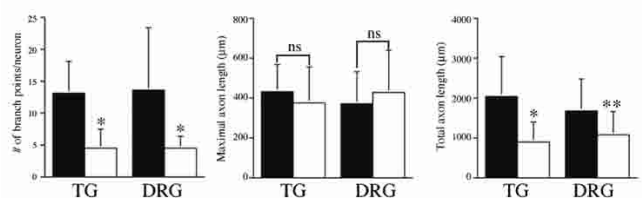
mond et al. 2000). The fact that Numb, and to a lesser extent $\mathrm{Nbl}$, has been shown to interact with the intracellular domain of Notch and antagonize the Notch in cell fate and dendritic branching led us to hypothesize that an increase in Notch activity may contribute to the sensory axon arborization phenotype in Wnt1Cre/+;fnb/ $f n b ; n b 1^{-/-}$mutants (Frise et al. 1996; Guo et al. 1996; Zhong et al. 1996, 1997; Berezovska et al. 1999; Sestan et al. 1999). To test this hypothesis, we used confocal microscopy to determine if loss of Numb and $\mathrm{Nbl}$ affected the distribution of Notch1 in cultured neurons.

We first examined the distribution of endogenous Numb, Nbl, and Notch1 in wild-type, untreated sensory neurons. To determine the subcellular localizations of these molecules, both DIC and lamin B immunofluorescence were obtained to delineate the nuclear and cytoplasmic boundaries (see Supplementary Fig. 1). By using a series of confocal images from different $z$ positions, we showed that Numb was present in discrete vesicular structures in cell body and axons (Fig. 5A,B2-E2). These results were consistent with the distribution of endogenous Numb in sensory neurons (cf. Fig. 1F,G). Many Numb-positive vesicles were colocalized with the endocytic marker Alexa fluor-conjugated wheat germ agglutinin (WGA) (Fig. 5B3-D3). To detect Notch1, we used an antibody that recognized the intracellular domain of Notch1. A range of Notch1 immunofluorescence was detected in cultured sensory neurons, from a diffuse low

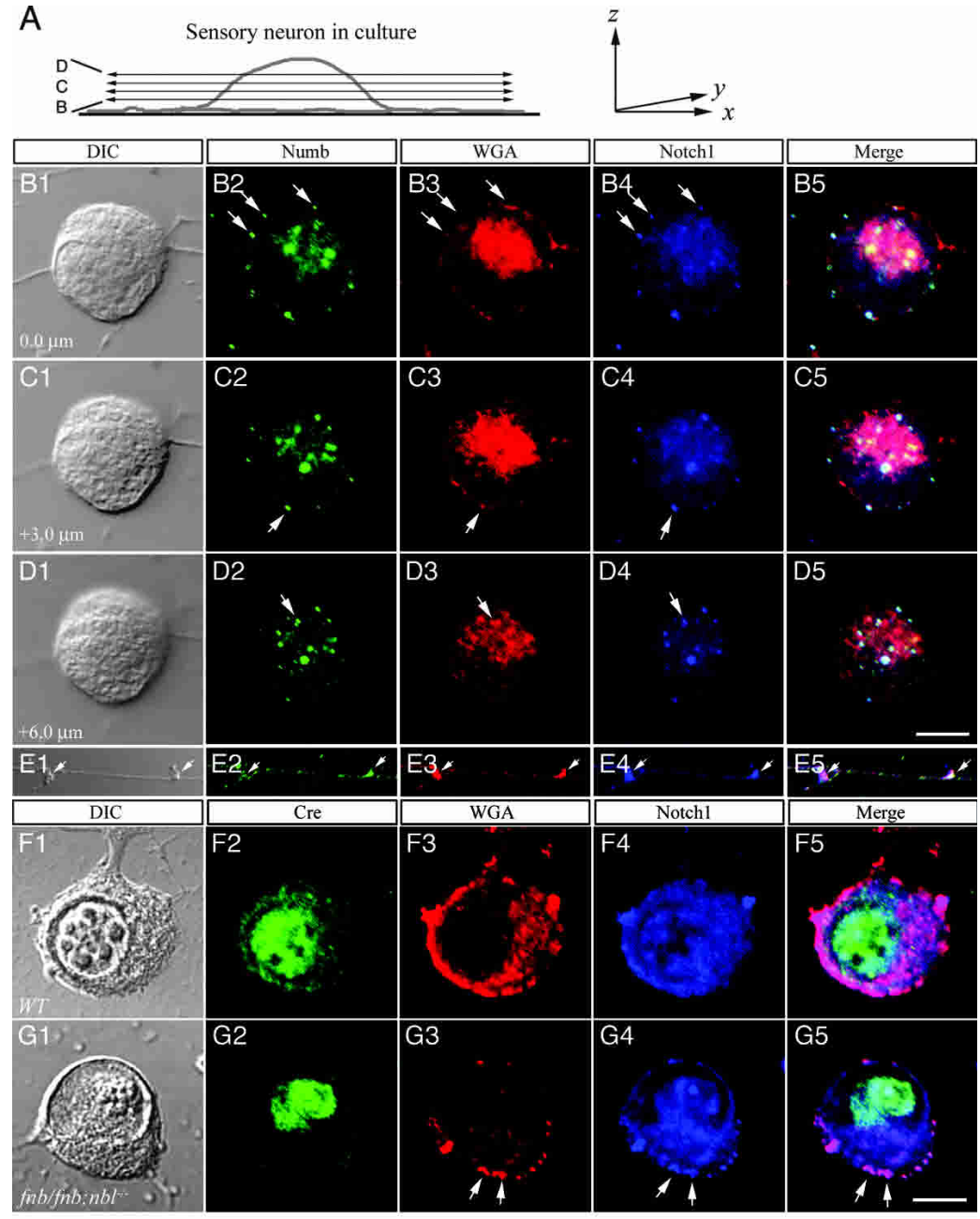

$\mathrm{H}$

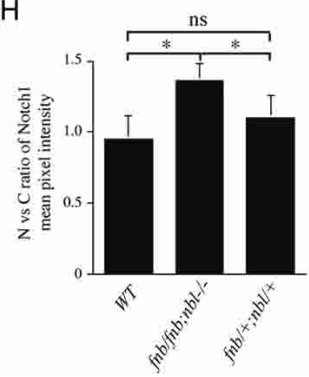

Figure 5. Loss of Numb and $\mathrm{Nbl}$ leads to a reduction in endocytic vesicles. $(A)$ Schematic diagram depicting the confocal planes obtained from a sensory neuron in culture, which are presented in $B-D$. $(B-E)$ Colocalization of Numb endosomal marker WGA and Notch1 ICD in the cytoplasm and axons of sensory neurons. Numb was present in endocytic vesicles and colocalized with Notch1 ICD in the wild-type sensory neurons (arrows in panels $B-D$ ). Interestingly, the colocalization of Numb and Notch1 ICD was also present in varicosities along sensory axons $(E) .(F, G)$ Deletion of $n u m b$, using HSV-iCre, in the nbl null background resulted in a marked reduction of WGApositive endocytic vesicles in the cytoplasm $(G 3)$, without affecting the presence of endocytic vesicles in the cell surface. The presence of intracytoplasmic Notch1 vesicles was reduced (G4). Most of the Notch1 protein was present on the cell surface (arrows in G4). DIC images in B1-G1 were presented to define the boundary of the nuclear membrane $(\mathrm{Q}, \mathrm{V}) .(H)$ Quantitative analyses of Notch immunofluorescent intensity in nucleus and cytoplasm. Ratio of nucleus-to-cytoplasm Notch was determined using Leica Confocal Software (for details, see Materials and Methods). Whereas expression of Cre recombinase did not alter the nucleus-to-cytoplasm ratio of Notch in either wild-type or $f n b /+; n b l /+$ neurons, it resulted in a modest increase of such ratio in $\mathrm{fnb} /$ fnb;nbl/nbl neurons. Student's $t$-test, ${ }^{\star} p<0.05$; ns indicates not significant. Bars: D5, $7 \mu \mathrm{m}$; G5, $5 \mu \mathrm{m}$. 
intensity presence with distinct vesicular structures (Fig. 5B-D) to slightly stronger staining in the nucleus and cytoplasm (Fig. 5F). The cause of this variation was unclear but could be related to differences in neuronal subtypes or the state of neuronal growth or differentiation. Regardless of the variable intensity, however, the ratio of Notch1 immunofluorescent intensity in the nucleus and cytoplasm was about equal (Fig. 5H). Interestingly, the distribution of Notch1 in sensory axons was remarkably similar to that of Numb and corresponded to 1-2 $\mu \mathrm{m}$ varicosities in DIC images (Fig. 5E). To investigate the nature of such axonal varicosities, we performed colabeling experiments using synaptophysin as a marker for synapses and WGA as an endocytic marker. Our results showed that Numb and Notch1 colocalized with WGA, but not with synaptophysin, in the axons (Fig. 5E; data not shown). Although the distribution of Nbl was similar to Numb, it appeared to be more diffuse in the cytoplasm and the staining of $\mathrm{Nbl}$ in the axonal varicosities was less intense compared with Numb (Supplementary Fig. 2).

The fact that Numb interacted with the endosomal protein $\alpha$-adaptin and affected trafficking of internalized receptors in nonneuronal cells led us to hypothesize that Numb may regulate internalization and transportation of Notch (Santolini et al. 2000). To test this hypothesis, we examined changes in endocytic process and the distribution of Notch1 in the absence of Numb and Nbl. We applied Alexa fluor-conjugated WGA to live neurons for $30 \mathrm{~min}$ before harvesting them for immunostaining ( $\mathrm{Ma}$ et al. 2002). Since translocation of Notch1 ICD into nucleus is required for Notch to regulate gene expression, we focused on the distribution of Notch1 in sensory neurons. Our results indicated that expression of Cre recombinase in $f n b / f n b ; n b l^{-/-}$neurons resulted in a reduction in the internalization of WGA+ vesicles on the cell surface and in the cytoplasm (Fig. 5, cf. F and G, arrows). Quantification of the relative signal intensity of Notch1 (using Leica Confocal Software, Leica Microsystem; for details, see Materials and Methods) in these neurons revealed a slight increase in the relative intensity of Notch1 ICD in the nucleus (Fig. 5G4,H). As a consequence, there was a modest, but significant increase in the nucleus-to-cytoplasm ratio of Notch1 in $\mathrm{fnb} / \mathrm{fnb} ; n b 1^{-1-}$, but not $f n b /+; n b 1^{-/-}$or wild-type neurons (Fig. $5 \mathrm{H}$ ).

\section{Overexpression of Numb leads to accumulation of Notch1 in abnormal endocytic/lysosomal vesicles}

Results in Figure 5 indicate that loss of Numb and $\mathrm{Nbl}$ influences intracellular trafficking of Notch1 without affecting its transport into the nucleus. Since previous results have indicated that the C-terminal tripeptide sequences of Numb, ${ }^{556}$ Asp-Pro-Phe and ${ }^{579}$ Asn-Pro-Phe, were required for the interaction with endocytic protein $\alpha$-adaptin (Santolini et al. 2000), we reasoned that deletion of such sequences potentially could perturb the effects of Numb in the endocytic processes. To test this hypothesis, we prepared HSV that express EGFP or EYFP fusion protein that contained wild-type Numb (EGFP- numb), mutant Numb lacking the C-terminal tripeptide sequences (EYFP-numb ${ }^{\Delta 57-593}$ ), or wild-type Nbl (EGFPnbl) (Fig. 6A). Whereas expression of EGFP did not affect the endocytosis of WGA (Fig. 6B), expression of EGFPnumb in wild-type sensory neurons resulted in a dramatic increase in the number and the size of WGA-positive vesicles, many of which also contained transferrinbiotin and Notch1 (Fig. 6C-E). Interestingly, the level of Notch1 showed a marked reduction in the nucleus of neuron expressing EGFP-numb (Fig. 6C-F). In addition to expressing the endocytic marker WGA and transferrin, most of these vesicles were also positive for late endosome/early lysosome marker LAMP1/2 and cathepsin D (Fig. 6E,F). In contrast, expression of EYFP-numb $b^{\Delta 557-593}$ or EGFP-nbl did not alter the number and distribution of WGA-positive endocytic vesicles, nor did it affect the distribution of Notch1 in the cytoplasm and nucleus (Fig. 6G; data not shown). The distribution of TrkA-positive vesicles was not affected in neurons expressing wildtype Numb (Fig. 6H), probably due to additional Numbindependent membrane receptor recycling mechanisms involving molecules, such as Pincher (Shao et al. 2002).

To provide a more quantitative analysis of Numb-induced changes, we determined the number of GFP-positive vesicles in neurons and the relative level of Notch1 ICD signal intensity in the cytoplasm and nucleus (Fig. 6I-K). Our data showed that, while most EGFP or EGFPnumb $b^{\Delta 57-593}$-expressing neurons showed no or very few GFP-positive vesicles in the cytoplasm, $>60 \%$ of neurons expressing EGFP-Numb contained more than five vesicles per cell (Fig. 6I). Analyses of Notch1 signal intensity revealed that the majority of EGFP-Numb neurons showed a significant decrease in the amount of Notch1 ICD in the nuclei (Fig. 6J). In contrast, the distribution of Notch1 in neurons expressing EGFP or EGFP-numb ${ }^{\Delta 557-593}$ was predominantly in the cytoplasm (Fig. 6J). As a consequence, the nuclear-to-cytoplasmic ratio of Notch1 signal was significantly reduced in neurons expressing EGFP-numb but not those expressing EGFP or EGFP-numb ${ }^{\Delta 557-593}$ (Fig. 6K).

Numb increases, whereas constitutively active Notch1 reduces, sensory axon arborization

To investigate the effects of Numb gain of function in axonal arborization, wild-type sensory neurons were infected with HSV expressing either EGFP or EGFP-numb $2 \mathrm{~h}$ after plating, cultured for $48 \mathrm{~h}$ and collected for analyses. In contrast to the dramatic reduction in axonal branching and total axon length in the absence of Numb and Nbl (Figs. 3, 4), neurons expressing EGFP-numb showed approximately two- to threefold increases in the number of branch points, maximal axon length, and total axonal length (Fig. 7B,D,E,F). The effects of Numb appeared to be similar in both NGF and NT3-responsive neurons from either trigeminal or dorsal root ganglia (Fig. 7E,F). Neurons expressing EYFP-numb ${ }^{\Delta 557-593}$, however, showed morphological features similar to those expressing EGFP (Fig. 7E,F), indicating that the 


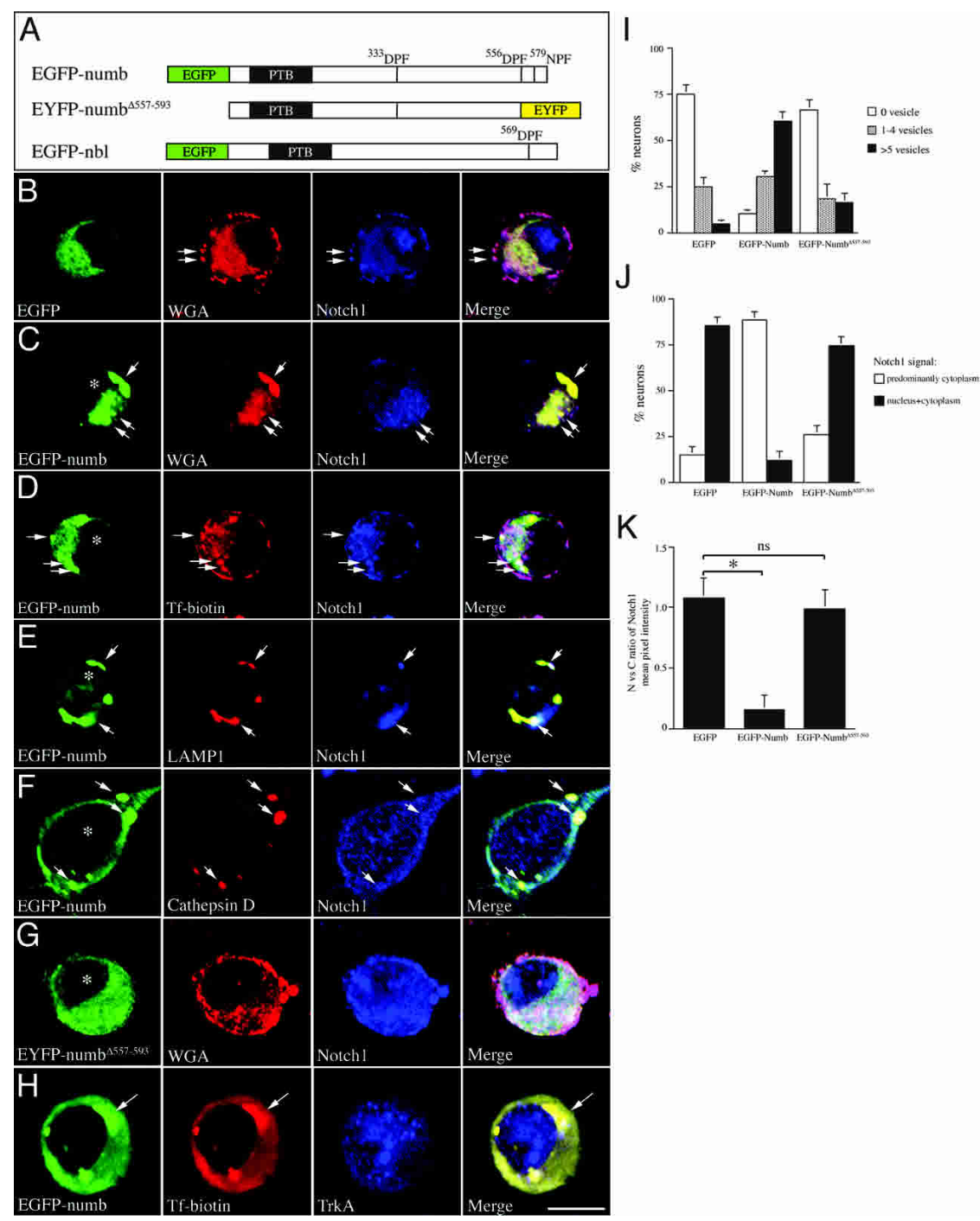

Figure 6. Overexpression of Numb, but not Numb mutant lacking $\alpha$-adaptin-interacting domain, leads to accumulation of markedly enlarged lysosomal vesicles and a reduction of Notch1 in the nucleus. (A) A Schematic diagram of constructs of wildtype Numb and $\mathrm{Nbl}$, and mutant Numb in which the tripeptide sequences interacting with $\alpha$-adaptin were deleted. (B) Expression of EGFP in sensory neurons had no effects in the distribution of endocytic vesicles and Notch1. Endocytic vesicles containing Notch1 were present in the cell surface (arrows) as well as in the cytoplasm. Significant amount of Notchl was detected in the nucleus. $(C-F)$ Expression of EGFP-numb led to accumulation of markedly enlarged vesicles in the cytoplasm. Most of these enlarged vesicles showed the properties of endosome $(C, D$, arrows indicate WGA and transferrin biotin-positivity in these vesicles, respectively) and lysosome $(E, F$, arrow shows the presence of LAMP1 and cathepsin D). Neurons expressing EGFP-numb showed a very low level of Notch1 in the cytoplasm and nucleus ( ${ }^{*}$ in $C-F$ ). Many of these neurons also showed accumulation of Notch1 in the enlarged vesicles (arrows in $C-F)$. (G) Deletion of the $\alpha$-adaptin-interacting tripeptide sequences in the $\mathrm{C}$ terminus of Numb abolished the effects of Numb to induce lysosome formation. $(H)$ The Numb-induced lysosomal vesicles showed accumulation of transferrin-biotin (arrows) without affecting the presence of TrkA vesicles. (I) Quantitative analyses of the percentage of neurons that contained more than one vesicle $(<1$ um diameter). Expression of EGFP-Numb, but not Numb mutant without the $\alpha$-adaptin-interaction domain, induced many markedly enlarged vesicles in the cytoplasm. $(J)$ Quantitative representation of the percentage of neurons that showed a predominant Notch1 staining in cytoplasm or in both nucleus and cytoplasm. $(K)$ The nuclear to cytoplasmic ratios of Notch1 were presented by measuring the mean pixel intensity using Leica Confocal Software. Paired Student's $t$-tests were used to compare the difference of Notch1 signal intensity between neurons expressing EGFP, EGFP-Numb, or EYFP-Numb ${ }^{\Delta 557-593}$. Bar: $H, 7 \mu \mathrm{m}$.

$\alpha$-adaptin-interacting domain in Numb was required for regulating axonal arborization.

By using both loss-of-function and gain-of-function analyses, we have shown that Numb and Nbl play important roles in sensory axon arborization, most probably through the regulation of Notch endocytosis. To determine if Notch can directly influence axon arborization in sensory neurons, we used a similar approach as in Figure 7 to express EGFP-tagged full-length Notch (NotchFL) and two constitutively active forms of Notch, Notch$\triangle \mathrm{EC}$, and Notch-IC (Berezovska et al. 1999), in sensory neurons cultured in the presence of NGF. Whereas Notch-FL showed a predominant membrane and cytoplasmic distribution in sensory neurons, Notch- $\Delta \mathrm{EC}$ and Notch-IC showed a predominant or exclusive localization in the nuclei, respectively (Fig. 8A-I). Consistent with the reported results in cortical neurons (Berezovska et al. 1999; Sestan et al. 1999; Redmond et al. 2000), both Notch- $\Delta \mathrm{EC}$ and Notch-IC significantly reduced the axon branch points, maximal axon length, and total axon length in sensory neurons $(n=25$ for each group, $p<0.01$; Fig. 8J-L). In contrast, Notch-FL had no effect on axon branch points and only minimal effects in reducing total and maximal axon length (Fig. 8J-L). Taken together, these data support the notion that, in contrast to Numb gain of function, accumulation of Notch in the nucleus negatively regulates sensory axon arborization.

\section{Discussion}

In this study, we reported that loss of Numb and $\mathrm{Nbl}$ in early murine sensory ganglia had no detectable effect on the development of sensory neurons. Instead, a severe reduction in collateral branching and total axon length 


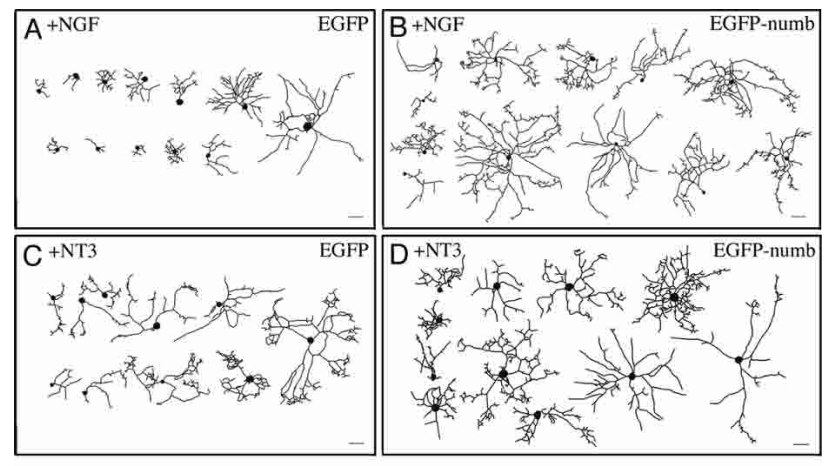

E NGF-responsive neurons
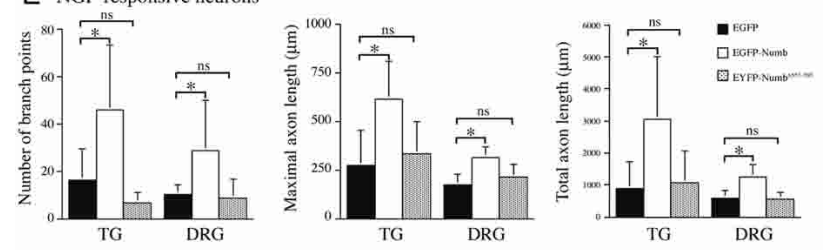

F NT3-responsive neurons
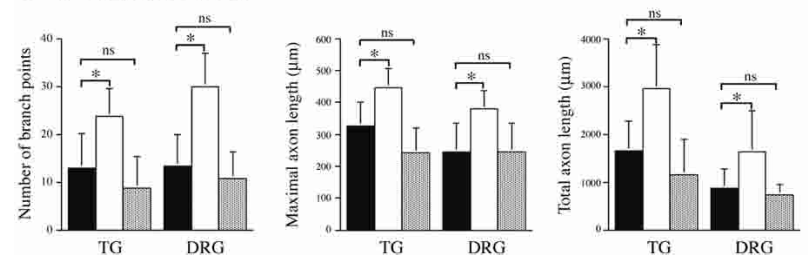

Figure 7. Overexpression of Numb promotes branching and lengthening of sensory axons. $(A-D)$ Depiction of representative images of neurons expressing EGFP $(A)$ or EGFP-numb $(B)$ grown in NGF or in the presence of NT3 $(C, D)$. Neurons were infected with HSV expressing EGFP or EGFP-numb, allowed to grow for $48 \mathrm{~h}$, and processed for image analyses. (E) Quantification of changes in axon branch points, maximal axon length, and total axon length in sensory neurons expressing EGFP, EGFP-numb, or EYFP-Numb ${ }^{\Delta 57-593}$. Expression of EGFP-numb, but not EYFP-Numb ${ }^{\Delta 57-593}$, drastically increased the number of collateral branches (left), maximal axon length (middle), and total axon length (right). (F) Expression of EGFP-numb also had a similar effects on the axon branching and length in NT3responsive neurons. Bars: $A-D, 100 \mu \mathrm{m}$. Paired Student's $t$-tests were used to for statistical analyses. ${ }^{\star} p<0.01$; ns indicates not significant.

was detected in sensory axons of the conditional mutants and in cultured neurons where Numb was deleted by Cre recombinase. The mechanisms by which Numb and $\mathrm{Nbl}$ affected axonal branching correlated with the endocytic processing of Notch1. In the absence of Numb and $\mathrm{Nbl}$, formation of the endocytic vesicles was severely reduced, with most neurons showing a modest increase in the nuclear-to-cytoplasmic ratio of Notch1. Conversely, overexpression of Numb led to increases in axonal branching and total axon length. Many of these neurons showed accumulation of Notch1 in markedly enlarged vesicles in the cytoplasm, with a concomitant reduction in nuclear Notch1. The Numb-induced cytoplasmic vesicles had characteristics of late endosomes and early lysosomes, suggesting a role for Numb in con- trolling a delicate balance between early and late endosomes in sensory neurons. Taken together, our data supported an unexpected, yet essential, function of the mouse numb homologs in sensory axon branching by regulating Notch1 in sensory neurons via the endocyticlysosomal pathways.

In contrast to the distinct roles of numb and $n b l$ in cortical neurogenesis (Zhong et al. 2000; Petersen et al. 2002, 2004; Li et al. 2003), functions of the vertebrate numb homologs in sensory neurogenesis are largely unknown. Our data indicate that deletion of numb and $n b l$ at the stages of active sensory neurogenesis does not affect the generation of sensory neurons or differentiation of neuronal subtypes (Fig. 2). The lack of an apparent phenotype in the sensory nervous system of numb conditional mutants raises the possibility that, in higher vertebrates, there may exist lineage-specific strategies that control fate determination and that mouse numb homologs may be dispensable in certain cell lineages. Indeed, transgenic overexpression of mouse numb using the Lck promoter reduced the expression of several Notch1 target genes without affecting the development, cell cycle regulation, or survival of thymocytes. Most importantly, although the Lck-numb transgenic mice showed expression of Numb in early stages of thymocyte development, such expression did not alter CD4/CD8 T cell fate specification (French et al. 2002).

While our findings support a lack of function of numb and $n b l$ in mouse sensory neurogenesis, these data are in direct contrast to that reported by Zilian and colleagues (2001), in which deletion of numb alone was reported to result in a severe reduction in the expression of neuronal markers NeuroD and NF160 and an up-regulation of glial marker ErbB3 in DRG of E10.5 embryos. Several possible explanations could account for such discrepancies. First, the effects of Numb in determining cell fate could occur before the closure of neural tube and emigration of neural crest cells. In this scenario, cell fate determination for cells in the sensory nervous system could be determined long before the emigration of neural crest from neural tube. Indeed, cell lineage tracing studies in mouse embryos have indicated that commitment to cranial and trunk neural crests is established before closure of the neural tube (Serbedzija et al. 1992, 1994). Since Wnt1Cre is only active after the closure of neural tube, this could in part account for the lack of neurogenesis phenotype in the sensory ganglia of Wnt1Cre/+; $n b / f n b ; n b 1^{-/-}$mice. Second, defects in the sensory ganglia could be an indirect consequence of a more general developmental defect in the mutant embryos. Indeed, numb mutants in the EIIaCre genetic background show defects in the developing blood vessels in head and trunk, failure in the closure of neural tube, and a near complete absence of the developing spinal cord at E10.5, which could have had an adverse effect on neural crest migration and the initial formation of sensory ganglia (Zilian et al. 2001).

In addition to the well-documented roles of Notch in cell fate determination, there is emerging evidence that Notch can also regulate the differentiation of post-mitotic neurons. In Drosophila, combinations of Notch and 

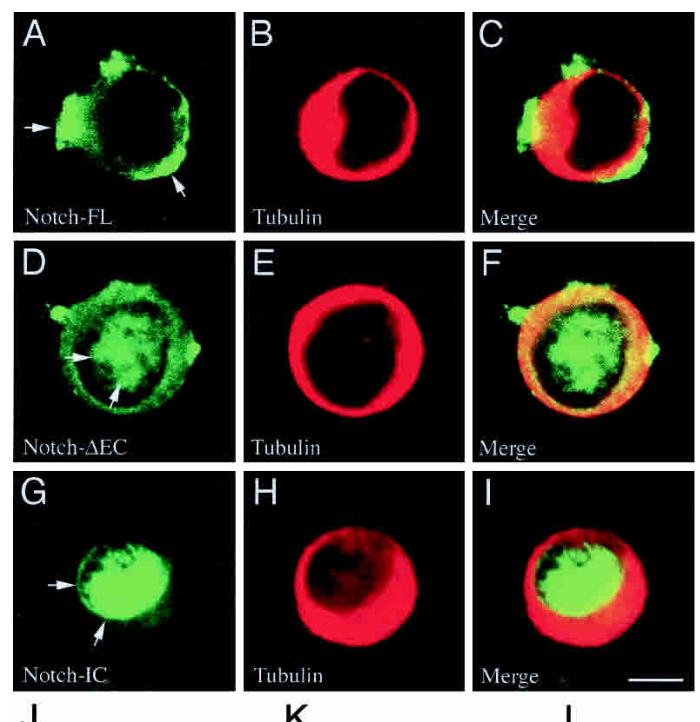

$\mathrm{J}$
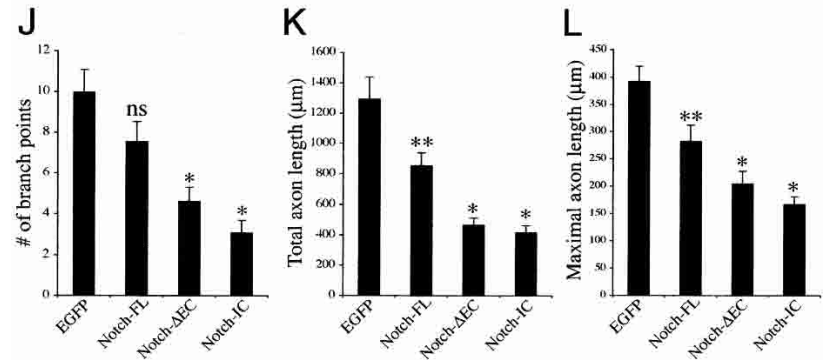

Figure 8. Constitutively active Notch negatively regulates sensory axon arborization. $(A-I)$ Constructs of full length (Notch-FL) and two constitutively active forms of Notch, Notch- $\Delta \mathrm{EC}$ and Notch-IC, are tagged with EGFP and expressed in cultured sensory neurons using HSV. $(A-C)$ When expressed in sensory neurons, Notch-FL shows a prominent presence on the cell membrane and in the cytoplasm, but not in the nucleus (arrows). In contrast, Notch- $\Delta \mathrm{EC}$ shows a predominant distribution in the nucleus, as well as on the cell membrane and in the cytoplasm $(D-F$, arrows), whereas Notch-IC shows a almost exclusive distribution in the nucleus ( $G-I$, arrows). Bar: $I, 5 \mu \mathrm{m}$. $(J-L)$ Expression of Notch-FL in sensory neurons has no effect on the number of axon branch points $(J)$, but induces a slight reduction in the total and maximal axon length $\left(K, L,{ }^{\star \star} 0.05<\right.$ $p<0.01, n=25$ for each group, one-way ANOVA). In contrast, both Notch- $\triangle \mathrm{EC}$ and Notch-IC induce significant reduction in axon branch points, total axon length and maximal axon length in sensory neurons. $\left({ }^{\star} p<0.01\right)$.

$a b 1$ mutations result in synergistic genetic interactions leading to lethality and defects in axonal outgrowth in the absence of any alteration in neuronal identity (Giniger 1998). It has been proposed that such regulatory roles of Notch could be mediated through interactions between the intracellular domain of Notch and PTB domain of Disabled (Dab), which can potentially recruit Abl to regulate organization of actin cytoskeleton via a sequential or parallel pathway. In mice, Notch1 protein can be detected in neurites and nuclei of embryonic and post-natal cortical neurons (Sestan et al. 1999; Redmond et al. 2000). Overexpression of Notch1 ICD inhibits neurite outgrowth and dendritic branching in cortical neurons and in cultured sensory neurons (Fig. 8). Most in- triguingly, the inhibitory effects of Notch1 ICD can be antagonized by Numb and Nbl, suggesting that key regulators of the Notch signaling pathway also play essential roles in maintaining neuronal differentiation (Berezovska et al. 1999; Sestan et al. 1999; Redmond et al. 2000).

In addition to these data, recent evidence also supports an independent function of $\mathrm{Numb}$ and $\mathrm{Nbl}$ in regulating neurite outgrowth. For instance, loss of numb has been shown to result in a drastic reduction of neurite length in cultured cortical neurons (Shen et al. 2002). Further-

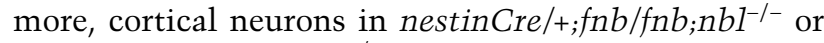

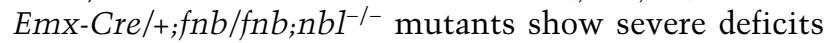
in dendritic growth and branching (Petersen et al. 2002, 2004; Li et al. 2003). Since the development of sensory neurons and spinal interneurons are not affected in

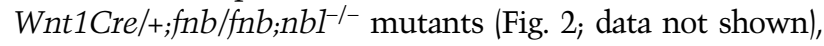
abnormalities in axonal growth and collateral branching in these mutants most likely are caused by cell-autonomous roles of numb and $n b l$ in regulating sensory axonal branching. Indeed, deletion of numb using HSV-iCre in sensory neurons from conditional mutants $\left(f_{n} b / f_{n} b\right.$; $\left.n b 1^{-/-}\right)$leads to a significant reduction in axonal branching and total length (Fig. 4).

How might Numb and $\mathrm{Nbl}$ regulate axonal growth and collateral branching in sensory neurons? Our results indicate that Numb and Notch1 show extensive colocalization in both axons and cytoplasm (Fig. 5). Consistent with our findings, Numb has been shown to colocalize with $\alpha$-adaptin and internalized EGFR and transferrin receptors in the endocytic vesicles in nonneuronal cells (Santolini et al. 2000). Furthermore, Numb interacts with the ear appendage of $\alpha$-adaptin via the two consecutive tripeptide sequences, Asp-Pro-Phe and Asn-ProPhe, in the C terminus (Fig. 6A; Santolini et al. 2000). Interestingly, these sequences are highly conserved in Numb and d-numb, suggesting that the interaction between Numb and $\alpha$-adaptin may be highly conserved in evolution (Fig. 6A; Zhong et al. 1997; Santolini et al. 2000). Indeed, d-numb also interacts with the ear domain of Drosophila $\alpha$-adaptin, and d-numb is required for asymmetric localization of $\alpha$-adaptin in SOP during mitosis (Berdnik et al. 2002). Most importantly, mutations in $\alpha$-adaptin lead to cell fate transformation phenotype that is similar to, albeit weaker than, numb mutants (Berdnik et al. 2002). Although epistatic analyses suggest that d-numb acts upstream of $\alpha$-adaptin and that Notch functions upstream or in parallel to $\alpha$-adaptin, detailed mechanisms of the interactions among these three molecules remain unclear.

The extensive colocalization of Numb and Notch 1 in endosomal vesicles of axons and cell body of sensory neurons (Fig. 5) suggests that Numb may regulate retrograde transport of Notch in post-mitotic neurons. Consistent with this notion, loss of numb results in a dramatic reduction in the number of endosomal vesicles (Fig. 5). In contrast, overexpression of Numb, but not $\mathrm{Nbl}$, leads to the formation of markedly enlarged vesicular structures with characteristics of late endosome and lysosome (Fig. 6C-F; data not shown). The ability of Numb to induce such abnormal changes in the endo- 
somal pathway requires the $\alpha$-adaptin-interacting domain in the $\mathrm{C}$ terminus as deletion of the two tripeptide sequences in Numb (EYFP-numb ${ }^{\Delta 557-593}$ ) completely abolishes this effect (Fig. 6G). Compared with Numb, $\mathrm{Nbl}$ contains only one tripeptide sequence $\left({ }^{569} \mathrm{DPF}\right)$ required for $\alpha$-adaptin interaction (Fig. 6A), is less abundant in the axons, and shows more diffuse distribution in the cytoplasm (Supplementary Fig. 2). Although overexpression of $\mathrm{Nbl}$ does not have similar effects as Numb, in loss-of-function analyses, Nbl shows partial compensatory effect in regulating axonal branching (Fig. 4C). It is possible that the redundancy between Numb and $\mathrm{Nbl}$ could be due to the fact that both proteins act in a sequential or parallel fashion to regulate the same pathway (see below).

Our data are consistent with the interpretation that Numb regulates endosomal pathway by controlling the delicate balance between the recycling of early endosome and the formation of late endosome/lysosome. One major function of the lysosomal pathway is to dampen signal transduction of membrane receptors by protein degradation (Brodsky et al. 2001; Sorkin and Von Zastrow 2002), which may contribute to the regulation of Notch1. In the absence of numb and $n b l$, sensory neurons show a modest increase of Notch1 in the nucleus (Fig. 5H). Since accumulation of nuclear Notch1 ICD has been shown to regulate gene expression and affect dendritic arborization, it is almost certainly true that a similar effect must regulate axonal branching in the sensory neurons (Berezovska et al. 1999; Sestan et al. 1999; Redmond et al. 2000). Conversely, overexpressing Numb leads to accumulation of Notch1 in the abnormally enlarged lysosomes with a concomitant reduction of Notch1 in the nucleus (Fig. 6). These findings suggest that at least two pathways are involved in regulating Notch signaling mechanisms. One is the endocytic-lysosomal pathway, in which Numb regulates endocytosis and degradation of Notch, while the other involves transport of Notch ICD to the nucleus through mechanisms that are poorly characterized at present time. Based on the distribution of Numb and Nbl in sensory neurons (Fig. 5), it is possible that these two proteins may regulate intracellular transport of Notch in a sequential or parallel mechanism. Our results complement the recent findings that endocytosis down-regulates LIN-12/Notch in Caenorhabditis elegans through a dileucine-containing sorting motif in the cytoplasmic domain of Notch (Shaye and Greenwald 2002). Moreover, it has also been shown that the Drosophila Notch ligand, Delta, is subject to ubiqutin ligase-dependent internalization and degradation, suggesting that the functions of Notch and Delta might be regulated through evolutionarily conserved endocytosis-mediated pathways (Lai et al. 2001; Pavlopoulos et al. 2001). Our results are consistent with the recent data that mammalian Numb promotes the ubiquitination of membrane-bound Notch1 through an E3 ligase-dependent mechanism, which leads to degradation of Notch1 ICD and loss of Notch-dependent transcriptional activation of Hes1 (McGill and McGlade 2003). The fact that Numb is involved in the endocytosis of Notch may have additional impacts on the cleavage of membrane-bound Notch. Indeed, recent reports indicate that Numb can also interact with the intracellular domain of $\beta$-amyloid precursor protein (APP) and such interaction is capable of inhibiting Notch signaling through $\gamma$-secretase-mediated pathways (Berezovska et al. 2000; Roncarati et al. 2002; Schroeter et al. 2003). Taken together, these data and our results underscore a previously unidentified function of Numb and $\mathrm{Nbl}$ in postmitotic neurons.

\section{Materials and methods}

\section{Animal husbandry}

Generation of conditional numb mutant mice and mice with null mutation in $n b l$ have been described in recent studies (Zhong et al. 2000; Petersen et al. 2002). Wnt1Cre transgenic mice were originally obtained from Dr. Andy McMahon (Harvard University, Cambridge, MA) (Chai et al. 2000; Jiang et al. 2000; Rico et al. 2002). Mice used for this study are maintained in both University of California San Francisco and San Francisco Veterans Administration Medical Center in accord with the guidelines of Institutional Animal Care Use Committee.

\section{Histology and immunohistochemistry}

Mouse embryos, from E9 to E15, were fixed with 4\% PFA in PBS. Newborn mice were anesthetized and perfused and fixed in $4 \%$ PFA. Cryostat sections were incubated with different combinations of primary antibodies and secondary antibodies. Cultured cells were fixed in 4\% PFA in PBS or ice-cold methanol and washed with PBS. Following fixation, tissue sections and cultures were blocked with $10 \mathrm{mM}$ Tris- $\mathrm{HCl}(\mathrm{pH} 7.5), 150 \mathrm{mM}$ $\mathrm{NaCl}, 0.4 \%$ Triton $\mathrm{X}-100,3 \%$ bovine serum albumin, and $10 \%$ calf serum for $1 \mathrm{~h}$ and incubated in primary antibody overnight in blocking solution. Antibodies used were as follows: rabbit anti-Neurofilament 150 (Chemicon, 1:1000), rabbit anti-TrkA (1:1000) (Huang et al. 1999a), rabbit anti-TrkB (1:300) (Huang et al. 1999a), goat anti-TrkC (1:300) (Huang et al. 1999a), rabbit anti-Parvalbumin (Swant, 1:1000), rabbit anti-Numb (1:300) (Zhong et al. 1996), rabbit anti-Numblike $(1: 1,000)$ (Zhong et al. 1997), rabbit anti-c-numb antibody (1:500) (Wakamatsu et al. 2000), goat anti-Notch1 (Santa Cruz Biotechnology, 1:500), mouse anti-Tubulin (Covance, 1:500), mouse anti-synaptophysin (Sigma, 1:500), mouse anti-Cre (BABCO, 1:500), and rabbit anti-Cre (BABCO, 1:3000). Secondary antibodies conjugated with Alexa series fluors, WGA-conjugated with Alexa 568, biotinylated transferrin, and avidin-rhodamine were from Molecular Probe. For DAB staining, biotinylated goat anti-rabbit IgG or goat anti-mouse IgG, and the $\mathrm{ABC}$ complex from Vectastain kit (Vector Laboratories) were used following the manufacturer's instructions (Ma et al. 2002). Cell count and BrdU injection and labeling have been described previously (Huang et al. 1999a,b). Image analyses were performed using the Leica TCS SP confocal microscope and Photoshop software.

\section{Construction of herpes viruses}

The construction of recombinant herpes viruses (HSVs) with high efficiency of neurotropism has been reported previously (Carlezon et al. 1997; Neve et al. 1997). Recombinant cDNA constructs of full-length $\mathrm{Numb}$ and $\mathrm{Nbl}$ fused with EGFP were generated using pEGFP-C2 plasmid (Clontech) and were sub- 
cloned into pHSVprpUC vector. Truncated version of Numb with deletion from amino acids 557-593 (numb ${ }^{\Delta 57-593}$ ) was generated by deletion the numb cDNA at the BamH1 site at amino acid 556 to remove the two tripeptide sequences at the C terminus (Santolini et al. 2000) and attached to the same restriction enzyme site in pEYFP-N2 plasmid. The resultant truncated construct was subcloned into pHSVprpUC. Codon improved Cre recombinase cDNA was generous provided by Drs. R. Sprengel (Max-Planck Institute for Medical Research, Heidelberg, Germany) and Louis Reichardt (University of California, San Francisco, CA) (Shimshek et al. 2002). HSVs carrying each of the aforementioned constructs were generated using the standardized methods (Neve et al. 1997).

\section{Sensory neuron culture}

Sensory neuron cultures were performed according to protocols that have been described in previous studies (Buchman and Davies 1993). Briefly, trigeminal ganglia were dissected from newborn mice, trypsinized, and triturated. Dissociated neurons were then plated on two-well chamber slides coated with polyDL-ornithine (0.5 mg/mL; Sigma) and laminin (20 $\mu \mathrm{g} / \mathrm{mL}$; GIBCO) at a density of 100-300 cells per well in defined F14 medium, containing $2 \mathrm{mM}$ glutamine, 0.35\% AlbuMax (GIBCO), $60 \mathrm{ng} /$ $\mathrm{mL}$ progesterone, $16 \mathrm{mg} / \mathrm{mL}$ putrescine, $400 \mathrm{ng} / \mathrm{mL}$ L-thyroxine, $38 \mathrm{ng} / \mathrm{mL}$ sodium selenite, and $340 \mathrm{ng} / \mathrm{mL}$ tri-iodothyronine. NGF was added to the culture at a final concentration of $10 \mathrm{ng} / \mathrm{mL}$. Because of the defined culture medium and low cell density ( 100-200 neurons were plated on two-well chamber slides), there were very few nonneuronal cells present (Buchman and Davies 1993). Sensory neurons were infected with HSV expressing EGFP-numb, EYFP-numb ${ }^{\Delta 557-593}$, or EGFP-nbl $3 \mathrm{~d}$ after plating and fixed 24 or $72 \mathrm{~h}$ after infection. For infection with HSV-iCre, sensory neurons were cultured from trigeminal ganglia of individual conditional mutant mouse that did not contain any Wnt1Cre transgene. After allowing sensory neurons to settle in chamber slides for $2 \mathrm{~h}$, the numbers of neurons in each chamber were determined and appropriate amounts of HSV-iCre virus were added to each chamber to achieve the infectivity of 100 moi. For Numb gain-of-function analyses, wildtype sensory neurons were infected with HSV expressing EGFP or EGFP-numb at $2 \mathrm{~h}$ after plating and cultured for additional 24 or $48 \mathrm{~h}$. For experiments on endocytosis, sensory neurons were incubated with Alexa fluor-conjugated WGA or transferrin-biotin for $30 \mathrm{~min}$ before the cells were harvested for immunostaining.

\section{Axon morphometry and analyses}

Unlike cortical or hippocampal neurons, nerve fibers emitting from the cell body of sensory neurons are uniformly positive for neurofilament and tubulin, and have been collectively referred to as axons and axon collaterals (Willis and Coggeshall 1991). Sensory neurons were fixed in either $4 \%$ PFA or ice-cold methanol and stained with TuJ1 antibody to reveal the axonal morphology. Images were captured by using a Hamamatsu CCD digital camera connected to a Zeiss fluorescent microscope driven by Openlab 3.0.1 Imaging Software (Improvision, Inc.). Whenever appropriate, multiple images were captured, and Camera Lucida drawings of neurons were performed to cover the entire axon length and branches. Images were then imported into the National Institutes of Health Image software program to measure the axon length. On average, images from at least 20 neurons were captured for statistical analyses. Each experiment was repeated at least three times and yielded comparable results. Data were present as mean \pm SEM. Most statistical analy- ses in Figures 4-8 were performed using paired Student's $t$-test, except for axon branch point analysis in Figure 4C, where one way ANOVA was applied to detect the difference among four genotypes followed by a Tukey's multiple comparison test. Statistical analyses were performed using graphPad Prism software (GraphPad Software Inc.).

\section{Confocal microscopy and image acquisition}

Images presented in Figures 5 and 6 were acquired by using the Leica TCS SP confocal microscope that was set at the same conditions for gain and pinhole. The laser intensity (measured as the PMT levels) for each fluorophor was kept within the linear range. Routinely, for EGFP and EGFP-tagged protein signals, PMT ranged from 650-700; for Numb and Nbl signals, PMT ranged from 680-745; for WGA or transferrin-biotin, PMT ranged from 650-700; for LAMP1 and cathepsin D signals, PMT ranged from 710-780; and for Notch1 signal, PMT ranged from 680-760. For comparison of Notch 1 signal intensity in the nucleus and cytoplasm (Figs. 5H, 6), we used the Leica Confocal Software (Leica Microsystem) and measured the mean pixel intensity for Notch1 in the nucleus and cytoplasm using handfree drawing program with the aid of corresponding DIC images to define the cellular boundaries. For structures to be defined as vesicles, the size must exceed $1 \mu \mathrm{m}$. This designation is based on the overall size of Numb-induced vesicles (Fig. 6).

\section{Acknowledgments}

We thank Dr. Andy McMahon for Wnt1Cre mice, Dr. Lou Reichardt for the Trk antibodies, Drs. Karla Zeitz and Allan Basbaum for help with behavioral analyses on Wnt1Cre/+; $f n b / f n b$ mice, Drs. Pier Paolo Di Fiore and Pece Salvatore for Numb antibody, Dr. Oskana Berezovska for Notch constructs, Ivy Hsieh for EM analyses on mutant mice, and Drs. Keli Hu and Zach Ma for sharing reagents. L.Y.J. and Y.N.J. are investigators of HHMI. This work has been supported by grants from UCSF Academic Senate and REAC, Merit Review Awards (VAMC), the Presidential Early Career Award for Scientists and Engineers, the Whitehall Foundation, and the NIH (NS44223) to E.J.H.

\section{References}

Anderson, D.J. 1999. Lineages and transcription factors in the specification of vertebrate primary sensory neurons. Curr. Opin. Neurobiol. 9: 517-524.

Arber, S., Ladle, D.R., Lin, J.H., Frank, E., and Jessell, T.M. 2000. ETS gene Er81 controls the formation of functional connections between group Ia sensory afferents and motor neurons. Cell 101: 485-498.

Berdnik, D., Torok, T., Gonzalez-Gaitan, M., and Knoblich, J.A. 2002. The endocytic protein $\alpha$-adaptin is required for numbmediated asymmetric cell division in Drosophila. Dev. Cell 3: 221-231.

Berezovska, O., McLean, P., Knowles, R., Frosh, M., Lu, F.M., Lux, S.E., and Hyman, B.T. 1999. Notch1 inhibits neurite outgrowth in postmitotic primary neurons. Neuroscience 93: 433-439.

Berezovska, O., Jack, P., McLean, C., Aster, J.C., Hicks, C., Xia, W., Wolfe, M.S., Kimberly, W.T., Weinmaster, G., Selkoe, D.J., et al. 2000. Aspartate mutations in presenilin and $\gamma$-secretase inhibitors both impair notchl proteolysis and nuclear translocation with relative preservation of notch 1 signaling. J. Neurochem. 75: 583-593.

Brodsky, F.M., Chen, C.Y., Knuehl, C., Towler, M.C., and Wakeham, D.E. 2001. Biological basket weaving: Formation 
and function of clathrin-coated vesicles. Annu. Rev. Cell. Dev. Biol. 17: 517-568.

Brown, A.G. 1981. Organization in the spinal cord: The anatomy and physiology of identified neurones. SpringerVerlag, New York.

Buchman, V.L. and Davies, A.M. 1993. Different neurotrophins are expressed and act in a developmental sequence to promote the survival of embryonic sensory neurons. Development 118: 989-1001.

Carlezon Jr., W.A., Boundy, V.A., Haile, C.N., Lane, S.B., Kalb, R.G., Neve, R.L., and Nestler, E.J. 1997. Sensitization to morphine induced by viral-mediated gene transfer. Science 277: 812-814.

Castaneda-Castellanos, D.R. and Kriegstein, A.R. 2004. Controlling neuron number: Does Numb do the math? Nat. Neurosci. 7: 793-794.

Chai, Y., Jiang, X., Ito, Y., Bringas Jr., P., Han, J., Rowitch, D.H., Soriano, P., McMahon, A.P., and Sucov, H.M. 2000. Fate of the mammalian cranial neural crest during tooth and mandibular morphogenesis. Development 127: 1671-1679.

French, M.B., Koch, U., Shaye, R.E., McGill, M.A., Dho, S.E., Guidos, C.J., and McGlade, C.J. 2002. Transgenic expression of numb inhibits notch signaling in immature thymocytes but does not alter $T$ cell fate specification. J. Immunol. 168: 3173-3180.

Frise, E., Knoblich, J.A., Younger-Shepherd, S., Jan, L.Y., and Jan, Y.N. 1996. The Drosophila Numb protein inhibits signaling of the Notch receptor during cell-cell interaction in sensory organ lineage. Proc. Natl. Acad. Sci. 93: 11925-11932.

Giniger, E. 1998. A role for Abl in Notch signaling. Neuron 20: 667-681.

Guillemot, F., Lo, L.C., Johnson, J.E., Auerbach, A., Anderson, D.J., and Joyner, A.L. 1993. Mammalian achaete-scute homolog 1 is required for the early development of olfactory and autonomic neurons. Cell 75: 463-476.

Guo, M., Jan, L.Y., and Jan, Y.N. 1996. Control of daughter cell fates during asymmetric division: Interaction of Numb and Notch. Neuron 17: 27-41.

Huang, E.J., Wilkinson, G.A., Farinas, I., Backus, C., Zang, K., Wong, S.L., and Reichardt, L.F. 1999a. Expression of Trk receptors in the developing mouse trigeminal ganglion: In vivo evidence for NT-3 activation of TrkA and TrkB in addition to TrkC. Development 126: 2191-2203.

Huang, E.J., Zang, K., Schmidt, A., Saulys, A., Xiang, M., and Reichardt, L.F. 1999b. POU domain factor Brn-3a controls the differentiation and survival of trigeminal neurons by regulating Trk receptor expression. Development 126: 2869-2882.

Jan, Y.N. and Jan, L.Y. 2001. Asymmetric cell division in the Drosophila nervous system. Nat. Rev. Neurosci. 2: 772-779.

Jiang, X., Rowitch, D.H., Soriano, P., McMahon, A.P., and Sucov, H.M. 2000. Fate of the mammalian cardiac neural crest. Development 127: 1607-1616.

Johnson, J.E., Birren, S.J., and Anderson, D.J. 1990. Two rat homologues of Drosophila achaete-scute specifically expressed in neuronal precursors. Nature 346: 858-861.

Klezovitch, O., Fernandez, T.E., Tapscott, S.J., and Vasioukhin, V. 2004. Loss of cell polarity causes severe brain dysplasia in Lgl1 knockout mice. Genes \& Dev. 18: 559-571.

Lai, E.C., Deblandre, G.A., Kintner, C., and Rubin, G.M. 2001. Drosophila neuralized is a ubiquitin ligase that promotes the internalization and degradation of $\delta$. Dev. Cell 1: 783-794.

Li, H.S., Wang, D., Kuo, C.T., Schonemann, M.D., Gorski, J.A., Jones, K.R., Jan, L.Y., and Jan, Y.N. 2003. Inactivation of Numb and Numblike in embryonic dorsal forebrain impairs neurogenesis and disrupts cortical morphogenesis. Neuron 40: $1105-1118$.
Ma, Q., Chen, Z., del Barco Barrantes, I., de la Pompa, J.L., and Anderson, D.J. 1998. neurogenin 1 is essential for the determination of neuronal precursors for proximal cranial sensory ganglia. Neuron 20: 469-482.

Ma, Q., Fode, C., Guillemot, F., and Anderson, D.J. 1999. Neurogenin 1 and neurogenin 2 control two distinct waves of neurogenesis in developing dorsal root ganglia. Genes \& Dev. 13: 1717-1728.

Ma, D., Zerangue, N., Raab-Graham, K., Fried, S.R., Jan, Y.N., and Jan, L.Y. 2002. Diverse trafficking patterns due to multiple traffic motifs in G protein-activated inwardly rectifying potassium channels from brain and heart. Neuron 33: 715729.

Markus, A., Zhong, J., and Snider, W.D. 2002. Raf and akt mediate distinct aspects of sensory axon growth. Neuron 35: 65-76.

McGill, M.A. and McGlade, C.J. 2003. Mammalian numb proteins promote Notch1 receptor ubiquitination and degradation of the Notch1 intracellular domain. J. Biol. Chem. 278: 23196-23203.

Morrison, S.J., Perez, S.E., Qiao, Z., Verdi, J.M., Hicks, C., Weinmaster, G., and Anderson, D.J. 2000. Transient Notch activation initiates an irreversible switch from neurogenesis to gliogenesis by neural crest stem cells. Cell 101: 499-510.

Neve, R.L., Howe, J.R., Hong, S., and Kalb, R.G. 1997. Introduction of the glutamate receptor subunit 1 into motor neurons in vitro and in vivo using a recombinant herpes simplex virus. Neuroscience 79: 435-447.

Patel, T.D., Jackman, A., Rice, F.L., Kucera, J., and Snider, W.D. 2000. Development of sensory neurons in the absence of NGF/TrkA signaling in vivo. Neuron 25: 345-357.

Pavlopoulos, E., Pitsouli, C., Klueg, K.M., Muskavitch, M.A., Moschonas, N.K., and Delidakis, C. 2001. Neuralized encodes a peripheral membrane protein involved in $\delta$ signaling and endocytosis. Dev. Cell 1: 807-816.

Perez, S.E., Rebelo, S., and Anderson, D.J. 1999. Early specification of sensory neuron fate revealed by expression and function of neurogenins in the chick embryo. Development 126: 1715-1728.

Petersen, P.H., Zou, K., Hwang, J.K., Jan, Y.N., and Zhong, W. 2002. Progenitor cell maintenance requires numb and numblike during mouse neurogenesis. Nature 419: 929-934.

Petersen, P.H., Zou, K., Krauss, S., and Zhong, W. 2004. Continuing role for mouse Numb and Numbl in maintaining progenitor cells during cortical neurogenesis. Nat. Neurosci. 7: 803-811.

Redmond, L., Oh, S.R., Hicks, C., Weinmaster, G., and Ghosh, A. 2000. Nuclear Notch1 signaling and the regulation of dendritic development. Nat. Neurosci. 3: 30-40.

Rico, B., Xu, B., and Reichardt, L.F. 2002. TrkB receptor signaling is required for establishment of GABAergic synapses in the cerebellum. Nat. Neurosci. 5: 225-233.

Roncarati, R., Sestan, N., Scheinfeld, M.H., Berechid, B.E., Lopez, P.A., Meucci, O., McGlade, J.C., Rakic, P., and D'Adamio, L. 2002. The $\gamma$-secretase-generated intracellular domain of $\beta$-amyloid precursor protein binds Numb and inhibits Notch signaling. Proc. Natl. Acad. Sci. 99: 7102-7107.

Santolini, E., Puri, C., Salcini, A.E., Gagliani, M.C., Pelicci, P.G., Tacchetti, C., and Di Fiore, P.P. 2000. Numb is an endocytic protein. J. Cell. Biol. 151: 1345-1352.

Schroeter, E.H., Ilagan, M.X., Brunkan, A.L., Hecimovic, S., Li, Y.M., Xu, M., Lewis, H.D., Saxena, M.T., De Strooper, B., Coonrod, A., et al. 2003. A presenilin dimer at the core of the $\gamma$-secretase enzyme: Insights from parallel analysis of Notch 1 and APP proteolysis. Proc. Natl. Acad. Sci. 100: 1307513080. 
Serbedzija, G.N., Bronner-Fraser, M., and Fraser, S.E. 1992. Vital dye analysis of cranial neural crest cell migration in the mouse embryo. Development 116: 297-307.

. 1994. Developmental potential of trunk neural crest cells in the mouse. Development 120: 1709-1718.

Sestan, N., Artavanis-Tsakonas, S., and Rakic, P. 1999. Contactdependent inhibition of cortical neurite growth mediated by notch signaling. Science 286: 741-746.

Shao, Y., Akmentin, W., Toledo-Aral, J.J., Rosenbaum, J., Valdez, G., Cabot, J.B., Hilbush, B.S., and Halegoua, S. 2002. Pincher, a pinocytic chaperone for nerve growth factor/TrkA signaling endosomes. J. Cell. Biol. 157: 679-691.

Shaye, D.D. and Greenwald, I. 2002. Endocytosis-mediated downregulation of LIN-12/Notch upon Ras activation in Caenorhabditis elegans. Nature 420: 686-690.

Shen, Q., Zhong, W., Jan, Y.N., and Temple, S. 2002. Asymmetric Numb distribution is critical for asymmetric cell division of mouse cerebral cortical stem cells and neuroblasts. Development 129: 4843-4853.

Shimshek, D.R., Kim, J., Hubner, M.R., Spergel, D.J., Buchholz, F., Casanova, E., Stewart, A.F., Seeburg, P.H., and Sprengel, R. 2002. Codon-improved Cre recombinase (iCre) expression in the mouse. Genesis 32: 19-26.

Soriano, P. 1999. Generalized lacZ expression with the ROSA26 Cre reporter strain. Nat. Genet. 21: 70-71.

Sorkin, A. and Von Zastrow, M. 2002. Signal transduction and endocytosis: Close encounters of many kinds. Nat. Rev. Mol. Cell. Biol. 3: 600-614.

Wakamatsu, Y., Maynard, T.M., and Weston, J.A. 2000. Fate determination of neural crest cells by NOTCH-mediated lateral inhibition and asymmetrical cell division during gangliogenesis. Development 127: 2811-2821.

Willis, W.D. and Coggeshall, R.E. 1991. Sensory mechanisms of the spinal cord. Plenum Press, New York.

Zhong, W., Feder, J.N., Jiang, M.M., Jan, L.Y., and Jan, Y.N. 1996. Asymmetric localization of a mammalian numb homolog during mouse cortical neurogenesis. Neuron 17: 43-53.

Zhong, W., Jiang, M.M., Weinmaster, G., Jan, L.Y., and Jan, Y.N. 1997. Differential expression of mammalian Numb, Numblike and Notch1 suggests distinct roles during mouse cortical neurogenesis. Development 124: 1887-1897.

Zhong, W., Jiang, M.M., Schonemann, M.D., Meneses, J.J., Pedersen, R.A., Jan, L.Y., and Jan, Y.N. 2000. Mouse numb is an essential gene involved in cortical neurogenesis. Proc. Natl. Acad. Sci. 97: 6844-6849.

Zilian, O., Saner, C., Hagedorn, L., Lee, H.Y., Sauberli, E., Suter, U., Sommer, L., and Aguet, M. 2001. Multiple roles of mouse Numb in tuning developmental cell fates. Curr. Biol. 11: 494-501. 


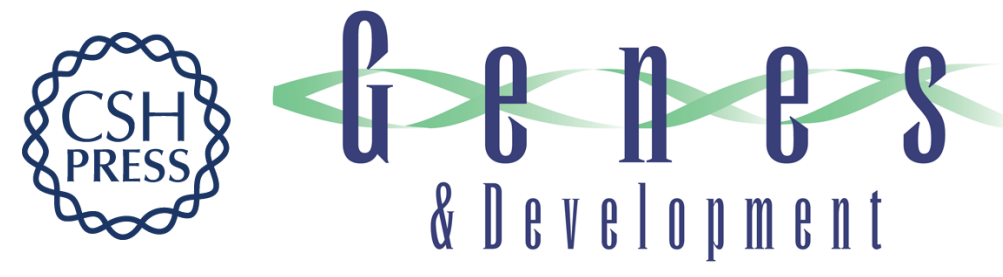

\section{Targeted deletion of numb and numblike in sensory neurons reveals their essential functions in axon arborization}

Eric J. Huang, Huashun Li, Amy A. Tang, et al.

Genes Dev. 2005, 19:

Access the most recent version at doi:10.1101/gad.1246005

Supplemental
Material http://genesdev.cshlp.org/content/suppl/2004/12/15/gad.1246005.DC1

References This article cites 53 articles, 22 of which can be accessed free at:

http://genesdev.cshlp.org/content/19/1/138.full.html\#ref-list-1

License

Email Alerting Receive free email alerts when new articles cite this article - sign up in the box at the top

Service right corner of the article or click here.

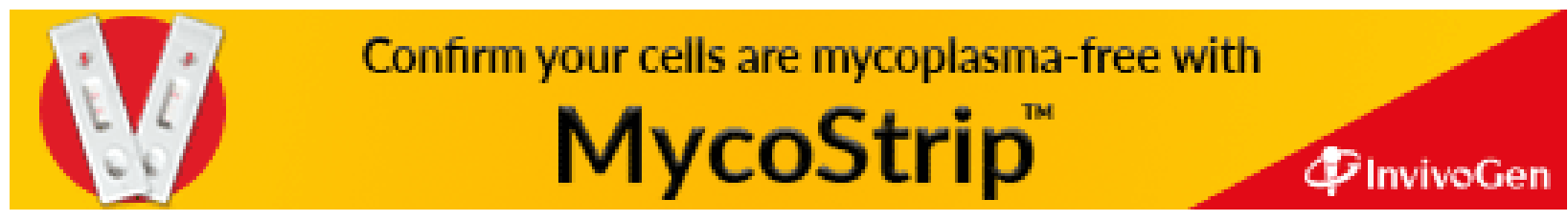

